\title{
ON THE MINIMAL EIGENVALUE OF THE LAPLACIAN OPERATOR FOR $p$-FORMS IN CONFORMALLY FLAT RIEMANNIAN MANIFOLDS ${ }^{1}$
}

\author{
DOMENICO PERRONE
}

\begin{abstract}
Let $(M, g)$ be a compact orientable conformally flat Riemannian manifold and ${ }^{p} \lambda_{1}$ the minimal eigenvalue of the Laplacian operator for $p$-forms. We prove that if there exists a positive constant $K$ such that $\rho>K g$, where $\rho$ is the Ricci tensor of $M$, then ${ }^{p} \lambda_{1} \geq K p(n-p+1) /(n-1)$ for each $p$, $1 \leq p \leq n / 2,(n=\operatorname{dim} M)$; moreover if the equality holds for some $p$ then $M$ is of constant curvature $\sigma=K /(n-1)$.
\end{abstract}

1. Introduction. Let $(M, g)$ be a compact orientable Riemannian manifold (connected, $C^{\infty}$ and $\operatorname{dim} M=n$ ) with metric tensor $g$. By $\Lambda^{p}(M), 0 \leq p \leq n$, we denote the space of the $p$-forms and by ${ }^{p} \Delta$ we denote the associated LaplaceBeltrami operator. It is given by

$$
{ }^{p} \Delta=d \delta+\delta d: \Lambda^{p}(M) \rightarrow \Lambda^{p}(M)
$$

where $d: \Lambda^{p}(M) \rightarrow \Lambda^{p+1}(M)$ is the exterior differential operator and $\delta: \Lambda^{p+1}(M) \rightarrow$ $\Lambda^{p}(M)$ is the exterior codifferential operator.

The spectrum of ${ }^{p} \Delta$, denoted by ${ }^{p} \operatorname{Spec}(M, g)$, has the following form: $\{0<$ $\left.{ }^{p} \lambda_{1} \ldots{ }^{p} \lambda_{1}<{ }^{p} \lambda_{2} \ldots{ }^{p} \lambda_{2}<{ }^{p} \lambda_{3} \ldots\right\} \rightarrow+\infty$ where each eigenvalue of ${ }^{p} \Delta$ is repeated as many times as its multiplicity.

For $\left(S^{n}, g_{0}\right)$, the sphere with the usual metric of positive constant sectional curvature $\sigma=K /(n-1)$, we have (E. Calabi):

$$
{ }^{0} \lambda_{1}=K n /(n-1) \text { and }{ }^{p} \lambda_{1}=K p(n-p+1) /(n-1) \text { for every } 1 \leq p \leq n / 2 \text {. }
$$

A theorem of A. Lichnerowicz [1, p. 179] states what follows: if there exists a positive constant $K$ such that $\rho \geq K g$, where $\rho$ denotes the Ricci tensor of $M$, then ${ }^{0} \lambda_{1} \geq K n /(n-1)$.

Under the same hypothesis of Lichnerowicz's theorem, Obata $[1$, p. 180] has proved that if ${ }^{0} \lambda_{1}=K n /(n-1)$, then $(M, g)$ is isometric to $\left(S^{n}, g_{0}\right)$.

In [2] S. Gallot and D. Meyer have obtained, under the hypothesis that the curvature operator $S$ satisfies $S \geq K$ with $K$ positive constant, the lower bound for ${ }^{p} \lambda_{1}$ and have discussed the case when ${ }^{p} \lambda_{1}$ takes the possible minimal value. For further topics related to estimates for eigenvalues of $\Delta$ see [6].

Now we assume our manifold to be conformally flat. Some results on the influence of the spectrum ${ }^{p} \operatorname{Spec}(M, g), p=0,1$, on the geometry of the conformally flat manifold have been studied in [5].

Received by the editors September 25, 1981.

1980 Mathematics Subject Classification. Primary 53C20; Secondary 53C25, 58G25.

${ }^{1}$ This work was carried out in the framework of the activities of the G.N.S.A.G.A. (C.N.R.Italy). 
In this paper we prove that if there exists a positive constant $K$ such that $\rho \geq$ $K g$, then ${ }^{p} \lambda_{1} \geq K p(n-p+1) /(n-1)$ for each $p, 1 \leq p \leq n / 2$, (cf. Theorem $3.2)$; moreover if the equality holds for some $p$ then $(M, g)$ is of constant curvature $\sigma=K /(n-1)$ (cf. Theorem 3.3).

2. Preliminaries. In the remainder of this paper, we denote by $g_{i j}, R_{j k h}^{i}, \rho_{i j}=$ $R_{i r j}^{r}$ the components of the metric, the curvature and the Ricci tensors respectively. By $C_{j k h}^{i}$ we denote the components of the Weyl conformal curvature tensor. We shall represent tensors by their components with respect to the natural base, and shall use the summation convention. $\nabla$ means the operator of covariant differentiation (with respect to the Riemannian connection) and $\tau=g^{i j} \rho_{i j}$ denotes the scalar curvature. For $\varphi, \psi \in \Lambda^{p}(M)$, the inner product $(\varphi, \psi)$, the lengths $\|\varphi\|$ and $\|\nabla \varphi\|$ are given by

$$
\begin{aligned}
(\varphi, \psi) & =\frac{1}{p !} \varphi_{i_{1} \cdots i_{p}} \psi^{i_{1} \cdots i_{p}}, \quad\|\varphi\|^{2}=(\varphi, \varphi), \\
\|\nabla \varphi\|^{2} & =\frac{1}{p !} \nabla_{r} \varphi_{i_{1} \cdots i_{p}} \nabla^{r} \varphi^{i_{1} \cdots i_{p}} .
\end{aligned}
$$

In $§ 3$ we shall use the following

LEMMA 2.1 [2, p. 270]. For every $p$-form in an n-dimensional Riemannian manifold, we have

$$
\|\nabla \varphi\|^{2} \geq \frac{1}{p+1}\|d \varphi\|^{2}+\frac{1}{n-p+1}\|\delta \varphi\|^{2} .
$$

3. The lower bound for ${ }^{p} \lambda_{1}$. In this section we prove the main results.

THEOREM 3.1. Let $(M, g)$ be a compact orientable Riemannian manifold of dimension $n \geq 4$. If there exists a constant $K>0$ such that $\rho_{i j} \geq K g_{i j}$, then

$$
{ }^{p} \lambda_{1} \geq \frac{K p(n-p+1)}{n-1}-\frac{H p(p-1)(n-p+1)}{2(n-p)} \quad \text { for each } p \text { with } 1 \leq p \leq \frac{n}{2} \text {, }
$$

where we put

$$
H=\operatorname{Sup}\left\{\frac{\left|C_{i j k h} \varphi^{i j} \varphi^{k h}\right|}{\varphi^{i j} \varphi_{i j}}: \varphi \in \Lambda^{2}(M)\right\}
$$

ProOF. For any $\varphi \in \Lambda^{p}(M)$ the following formula (cf. [4, p. 3]) holds:

$$
\frac{1}{2}^{0} \Delta\left(\|\varphi\|^{2}\right)=\left(\varphi,{ }^{p} \Delta \varphi\right)-\|\nabla \varphi\|^{2}-Q_{p}(\varphi)
$$

where $Q_{p}(\varphi)$ is the quadratic form defined by

$$
Q_{p}(\varphi)=\frac{1}{(p-1) !}\left[\rho_{i j} \varphi^{i i_{2} \cdots i_{p}} \varphi^{j i_{2} \cdots i_{p}}-\frac{p-1}{2} R_{i j k h} \varphi^{i j i_{3} \cdots i_{p}} \varphi^{k h i_{3} \cdots i_{p}}\right] .
$$

Since the Weyl's conformal curvature tensor is given by

$$
\begin{aligned}
R_{i j k h}= & C_{i j k h}+\frac{1}{n-2}\left(\rho_{j h} g_{i k}+\rho_{i k} g_{j h}-\rho_{j k} g_{i h}-\rho_{i h} g_{j k}\right) \\
& -\frac{\tau}{(n-1)(n-2)}\left(g_{i k} g_{j h}-g_{i h} g_{j k}\right),
\end{aligned}
$$


we have the following formula:

$$
\begin{aligned}
R_{i j k h} \varphi^{i j i_{3} \cdots i_{p}} \varphi_{i_{3} \cdots i_{p}}^{k h}= & \frac{4}{n-2} \rho_{i j} \varphi^{i i_{2} \cdots i_{p}} \varphi_{i_{2} \cdots i_{p}}^{j} \\
& -\frac{2 \tau}{(n-1)(n-2)} \varphi^{i_{1} \cdots i_{p}} \varphi_{i_{1} \cdots i_{p}}+C_{i j k h} \varphi^{i j i_{3} \cdots i_{p}} \varphi_{i_{3} \cdots i_{p}}^{k h}
\end{aligned}
$$

Hence

$$
\begin{aligned}
Q_{p}(\varphi)=\frac{1}{(p-1) !}[ & \frac{n-2 p}{n-2} \rho_{i j} \varphi^{i i_{2} \cdots i_{p}} \varphi_{i_{2} \cdots i_{p}}^{j} \\
& \quad+\frac{(p-1) \tau}{(n-1)(n-2)} \varphi^{i_{1} \cdots i_{p}} \varphi_{i_{1} \cdots i_{p}} \\
& \left.\quad-\frac{p-1}{2} C_{i j k h} \varphi^{i j i_{3} \cdots i_{p}} \varphi_{i_{3} \cdots i_{p}}^{k h}\right]
\end{aligned}
$$

On the other hand, using the hypothesis of the theorem, fixing a point in the manifold and taking a coordinate system, we have

$$
\begin{gathered}
\rho_{i j} \varphi^{i i_{2} \cdots i_{p}} \varphi_{i_{2} \cdots i_{p}}^{j} \geq K g_{i j} \varphi^{i i_{2} \cdots i_{p}} \varphi_{i_{2} \cdots i_{p}}^{j}=p ! K\|\varphi\|^{2}, \quad \tau=g^{i j} \rho_{i j} \geq n K, \\
-C_{i j k h} \varphi^{i j i_{3} \cdots i_{p}} \varphi_{i_{3} \cdots i_{p}}^{k h} \geq-H \varphi^{i j i_{3} \cdots i_{p}} \varphi_{i j i_{3} \cdots i_{p}}=-p ! H\|\varphi\|^{2} .
\end{gathered}
$$

Consequently, from (3.2), we have

$$
\begin{aligned}
Q_{p}(\varphi) & \geq p\left[\frac{n-2 p}{n-2} K+\frac{n(p-1)}{(n-1)(n-2)} K-\frac{p-1}{2} H\right]\|\varphi\|^{2} \\
& =p\left[\frac{n-p}{n-1} K-\frac{p-1}{2} H\right]\|\varphi\|^{2}
\end{aligned}
$$

at the fixed point in this coordinate system. But it is evident that this is valid also at all points and in all coordinate systems.

Now if $\varphi$ is an eigenform for ${ }^{p} \Delta$ corresponding to the eigenvalue ${ }^{p} \lambda_{1}$, i.e. ${ }^{p} \Delta \varphi=$ ${ }^{p} \lambda_{1} \varphi$ with $\|\varphi\|^{2} \neq 0$, by integration of (3.1) over $M$ with respect to the volume element $d v$ we have

$$
{ }^{p} \lambda_{1} \int_{M}\|\varphi\|^{2} d v=\int_{M}\left\|\nabla \varphi^{2}\right\| d v+\int_{M} Q_{p}(\varphi) d v .
$$

Moreover by integration of the inequality in Lemma 2.1, since $p \leq n-p$, we have

$$
\begin{aligned}
\int_{M}\|\nabla \varphi\| d v & \geq \frac{1}{n-p+1} \int_{M}\left(\|d \varphi\|^{2}+\|\delta \varphi\|^{2}\right) d v \\
& =\frac{1}{n-p+1} \int_{M}\left(\varphi,{ }^{p} \Delta \varphi\right) d v=\frac{{ }^{p} \lambda_{1}}{n-p+1} \int_{M}\|\varphi\|^{2} d v
\end{aligned}
$$

Finally by (3.4), because of (3.3) and (3.5), it follows that

$$
{ }^{p} \lambda_{1} \frac{n-p}{n-p+1} \int_{M}\|\varphi\|^{2} d v \geq p\left[\frac{n-p}{n-1} K-\frac{p-1}{2} H\right] \int_{M}\|\varphi\|^{2} d v .
$$

This completes the proof of Theorem 3.1. 
THEOREM 3.2. Let $(M, g)$ be a compact orientable conformally flat Riemannian manifold of dimension $n \geq 4$. If there exists a constant $K>0$ such that $\rho_{i j} \geq$ $K g_{i j}$, then

$$
{ }^{p} \lambda_{1} \geq \frac{p(n-p+1)}{(n-1)} K \quad \text { for each } p, 1 \leq p \leq \frac{n}{2} .
$$

ProOF. This follows from Theorem 3.1 since for a conformally flat Riemannian manifold the tensor $C_{i j k h}$ is a zero tensor.

REMARK 3.1. For $p=1$ Theorem 3.2 holds also without the hypothesis that $(M, g)$ be conformally flat.

REMARK 3.2. The Hodge star operator * defines an isomorphism $\Lambda^{p}(M) \rightarrow$ $\Lambda^{n-p}(M)$; besides if $\varphi$ is a $p$-form such that ${ }^{p} \Delta \varphi=\lambda \varphi$ then $* \varphi$ is a $(n-p)$-form such that ${ }^{n-p} \Delta(* \varphi)=\lambda(* \varphi)$.

Consequently ${ }^{p} \lambda_{1}={ }^{n-p} \lambda_{1}$, and thus from Theorem 3.2 we have

$$
{ }^{p} \lambda_{1}={ }^{n-p} \lambda_{1}=\frac{(n-p)(p+1)}{(n-1)} K \quad \text { for every } p, \frac{n}{2} \leq p \leq n-1 \text {. }
$$

Now we prove a theorem analogous to the theorem of Obata.

THEOREM 3.3. In the same hypothesis of Theorem 3.2, if

or

$$
{ }^{p} \lambda_{1}=\frac{p(n-p+1)}{n-1} K \text { for some } p, 1 \leq p \leq \frac{n}{2},
$$

$$
{ }^{p} \lambda_{1}=\frac{(n-p)(p+1)}{(n-1)} K \text { for some } p, \frac{n}{2} \leq p \leq n-1,
$$

then $(M, g)$ is a Riemannian manifold of constant sectional curvature $\sigma=$ $K /(n-1)$.

PrOOF. We denote by $E_{\lambda}$ the eigenspace of $p$-forms corresponding to $\lambda$. If we put

where

$$
{ }^{p} \lambda_{1}^{\prime}=\inf \left\{\lambda / E_{\lambda}^{\prime} \neq\{0\}\right\} \text { and }{ }^{p} \lambda_{1}^{\prime \prime}=\inf \left\{\lambda / E_{\lambda}^{\prime \prime} \neq\{0\}\right\},
$$

$$
E_{\lambda}^{\prime}=\left\{\varphi \in E_{\lambda} / d \varphi=0\right\} \text { and } E_{\lambda}^{\prime \prime}=\left\{\varphi \in E_{\lambda} / \delta \varphi=0\right\},
$$

by a proof similar to that of Theorem 3.1, it is easy to see that

$$
{ }^{p} \lambda_{1}^{\prime} \geq \frac{p(n-p+1)}{n-1} K \quad \text { and } \quad{ }^{p} \lambda_{1}^{\prime \prime} \geq \frac{(p+1)(n-p)}{n-1} K \quad(1 \leq p \leq n) .
$$

Moreover for the decomposition of Hodge-deRham we have $E_{\lambda}=E_{\lambda}^{\prime} \oplus E_{\lambda}^{\prime \prime}$ and hence ${ }^{p} \lambda_{1}=\inf \left\{{ }^{p} \lambda_{1}^{\prime},{ }^{p} \lambda_{1}^{\prime \prime}\right\}$.

On the other hand

$$
{ }^{p} \lambda_{1} \geq \frac{p(n-p+1)}{n-1} K \text { for } 1 \leq p \leq \frac{n}{2}
$$

and

$$
{ }^{p_{1}} \geq \frac{(p+1)(n-p)}{n-1} K \quad \text { for } \frac{n}{2} \leq p \leq n-1 .
$$

Therefore ${ }^{p} \lambda_{1}={ }^{p} \lambda_{1}^{\prime}$ for $1 \leq p \leq n / 2$, and ${ }^{p} \lambda_{1}={ }^{p} \lambda_{1}^{\prime \prime}$ for $n / 2 \leq p \leq n-1$. Thus if we consider an eigenform $\varphi$ corresponding to ${ }^{p} \lambda_{1}=(p(n-p+1) /(n-1)) K$, $1 \leq p \leq n / 2$, then it is closed i.e. $d \varphi=0$. 
In particular for $p=1$ we have

$$
{ }^{0} \Delta \delta \varphi=\delta^{1} \Delta \varphi=\frac{n K}{n-1} \delta \varphi \quad \text { with } \delta \varphi \neq 0,
$$

i.e. $\delta \varphi$ is an eigenfunction associated to the eigenvalue $n K /(n-1)$. From Obata's theorem it follows that $M$ is of constant sectional curvature $\sigma=K /(n-1)$.

Now we suppose $1<p \leq n / 2$. Because ${ }^{p} \lambda_{1}=(p(n-p+1) /(n-1)) K,(3.4)$ becomes

$$
\int_{M}\left(\|\nabla \varphi\|^{2}-\frac{p K}{n-1}\|\varphi\|^{2}\right) d v+\int_{M}\left(Q_{p}(\varphi)-\frac{p(n-p)}{n-1} K\|\varphi\|^{2}\right) d v=0
$$

On the other hand

$$
\|\nabla \varphi\|^{2} \geq \frac{1}{n-p+1}\|\delta \varphi\|^{2}=\frac{1}{n-p+1}\left(\varphi,{ }^{p} \Delta \varphi\right)=\frac{p K}{n-1}\|\varphi\|^{2}
$$

and

$$
\begin{aligned}
Q_{p}(\varphi) & =\frac{n-2 p}{(p-1) !(n-2)} \rho_{i j} \varphi^{i i_{2} \cdots i_{p}} \varphi_{i_{2} \cdots i_{p}}^{j}+\frac{p(p-1) \tau}{(n-1)(n-2)}\|\varphi\|^{2} \\
& \geq \frac{p(n-2 p)}{n-2} K\|\varphi\|^{2}+\frac{n p(p-1)}{(n-1)(n-2)} K\|\varphi\|^{2} \\
& =\frac{p(n-p)}{n-1} K\|\varphi\|^{2} .
\end{aligned}
$$

Therefore, from (3.6), we have

$$
\|\nabla \varphi\|^{2}=\frac{1}{n-p+1}\|\delta \varphi\|^{2} \quad \text { and } \quad Q_{p}(\varphi)=\frac{p(n-p)}{n-1} K\|\varphi\|^{2}
$$

In particular we have

$$
p(p-1)(\tau-n K)\|\varphi\|^{2}=0 \quad \text { with } p>1 \text { and }\|\varphi\|^{2} \neq 0,
$$

for which $\tau=n K=$ const.

Summing up we have that $M$ is a compact orientable conformally flat Riemannian manifold with constant scalar curvature and with positive Ricci curvature. Using Theorem A of Tani [7], we have that $M$ is of constant sectional curvature $\sigma=K /(n-1)$ (in fact $n(n-1) \sigma=\tau=n K)$.

For Remark 3.2, Theorem 3.3 holds also if ${ }^{p} \lambda_{1}=(n-p)(p+1) K /(n-1)$ for some $p, n / 2 \leq p \leq n-1$. Q.E.D.

The following corollary is a consequence of Theorem 3.3 and of Theorem 7.10 of [3, p. 265].

COROLlARY 3.1. In the same hypothesis of Theorem 3.3, if $M$ is simply connected, then it is isometric to the sphere $\left(S^{n}, g_{0}\right)$ of positive constant sectional curvature $K /(n-1)$.

REMARK 3.3. For manifolds of dimension $n=3$, that are not conformally flat, Theorem 3.2 is again valid since for every 3-dimensional Riemannian manifold the tensor $C_{i j k h}$ is a zero tensor. In dimension 3 also Theorem 3.3 is valid without the hypothesis that $M$ be conformally flat. In fact for $p=1$ (as seen already) Theorem 3.3 follows from Obata's theorem and, for $p=2,{ }^{2} \lambda_{1}={ }^{n-1} \lambda_{1}={ }^{1} \lambda_{1}$. 


\section{REFERENCES}

1. M. Berger, P. Gauduchon and E. Mazet, Le spectre d'une variété Riemannienne, Lecture Notes in Math., vol. 194, Springer-Verlag, Berlin and New York, 1971.

2. S. Gallot and D. Meyer, Opérateur de courbure et laplacien des formes différentielles d'une variété Riemannienne, J. Math. Pures Appl. 54 (1975), 259-284.

3. S. Kobsyashi and K. Nomizu, Foundations of differential geometry, vol. I, Interscience, New York, 1963.

4. A. Lichnerowicz, Géométrie des groupes de transformations, Dunod, Paris, 1958.

5. D. Perrone, Varietd conformemente piatte e geometria spettrale, Riv. Mat. Univ. Parma (4) 8 (1982) (preprint in Quaderni Ist. Mat. Univ. Lecce Q.20, 1980).

6. U. Simon and H. Wissner, Geometry of the Laplace operator, Technische Universität Berlin, Preprint Reihe Mathematik, No. 73, 1980.

7. M. Tani, On a compact conformally flat space with positive Ricci curvature, Tôhoku Math. J. 19 (1967), 227-231.

Istituto Di MATEMATICA, UNIVERSitÀ di LECCE, LECCE, ITALY 